gene are correlated with body weight. If it turns out that $\alpha-\mathrm{MSH}_{1-13}$ is the principal substrate of PRCP, then pharmacological inhibition of its action in the brain could help reduce appetite and weight gain; Wallingford et al. (8) demonstrated the efficacy of this strategy by injecting inhibitors directly into the brain, but brain-penetrating drugs would need to be developed to be clinically useful.

Address correspondence to: Richard D. Palmiter, Department of Biochemistry and Genome Sciences, Health Sciences Building, Room J-661F, Box 357370, University of Washington, 1959 Pacific Avenue, Seattle, Washington 98195, USA. Phone: (206) 543-6064; Fax: (206) 543-0858; E-mail: palmiter@u.washington.edu.

1. Smart, J.L., Tolle, V., and Low, M.J. 2006. Glucocorticoids exacerbate obesity and insulin resistance in neuron-specific proopiomelanocortin-deficient mice. J. Clin. Invest. 116:495-505.
2. Krude, H., et al. 1998. Severe early-onset obesity, adrenal insufficiency and red hair pigmentation caused by POMC mutations in humans. Nat. Genet. 19:155-157.

3. Coll, A.P., et al. 2004. Proopiomelanocortin and energy balance: insights from human and murine genetics. J. Clin. Endocrinol. Metab. 89:2557-2562.

4. Faroogi, I.S. 2008. Monogenetic human obesity. Front. Horm. Res. 36:1-11.

5. Saper, C.B., Chou, T.C., and Elmquist, J.K. 2002. The need to feed: homeostatic and hedonic control of eating. Neuron. 36:199-211.

6. Cone, R.D. 2005. Anatomy and regulation of the central melanocortin system. Nat. Neurosci. 8:571-578.

7. Morton, G.J., Cummings, D.E., Baskin, D.G., Barsh, G.S., and Schwartz, M.W. 2006. Central nervous system control of food intake and body weight. Nature. 443:289-295.

8. Wallingford, N., et al. 2009. Prolylcarboxypeptidase regulates food intake by inactivating $\alpha-\mathrm{MSH}$ in rodents. J. Clin. Invest. 119:2291-2303.

9. Prichard, L.E., Turnbull, A.V., and White, A. 2002. Pro-opiomelanocortin processing in the hypothalamus: impact on melanocortin signaling and obesity. J. Endocrinol. 172:411-421.

10. Jackson, R.S., et al. 1997. Obesity and impaired prohormone processing associated with mutations in the human prohormone convertase 1 gene. Nat. Genet. 16:303-306.

11. Rouillé, Y., et al. 1995. Proteolytic processing mechanisms in the biosynthesis of neuroendocrine peptides: the subtilisin-like proprotein convertases. Front. Neuroendocrinol. 16:322-361.

12. Naggert, J.K., et al. 1995. Hyperproinsulinaemia in obese fat/fat mice associated with a carboxypeptidase E mutation which reduces enzyme activity. Nat. Genet. 10:135-142.

13. Skidgel, R.A., and Erdos, E.G. 1998. Cellular carboxypeptidases. Immunol. Rev. 161:129-141.

14. Schmaier, A.H. 2003. The kallikrein-kinin and the renin-angiotensin systems have a multilayered interaction. Am. J. Physiol. Regul. Integr. Comp. Physiol. 285:R1-R13.

15. Diament, A.L., and Warden, C.H. 2004. Multiple linked mouse chromosome 7 loci influence body fat mass. Int. J. Obes. Relat. Metab. Disord. 28:199-210.

16. McCarthy, J.J., et al. 2003. Evidence for substantial effect modification by gender in a large-scale genetic association study of the metabolic syndrome among coronary heart disease patients. Hum. Genet. 114:87-98.

17. Wilkinson, C.W. 2006. Roles of acetylation and other post-translational modifications in melanocortin function and interactions with endorphins. Peptides. 27:453-471.

18. Guo, L., Munzberg, H., Stuart, R.C., Nillni, E.A., and Bjorbaek, C. 2004. N-acetylation of hypothalamic alpha-melanocyte-stimulating hormone and regulation by leptin. Proc. Natl. Acad. Sci. U. S. A. 101:11797-11802.

\title{
Engaging the lysosomal compartment to combat B cell malignancies
}

\author{
Kirsten Grønbæk ${ }^{1}$ and Marja Jäättelä ${ }^{2}$ \\ ${ }^{1}$ Department of Hematology, Rigshospitalet, Copenhagen, Denmark. ${ }^{2}$ Apoptosis Department and Centre for Genotoxic Stress Response, \\ Institute for Cancer Biology, Danish Cancer Society, Copenhagen, Denmark.
}

The combination of rituximab, a type I anti-CD20 $\mathrm{mAb}$, with conventional chemotherapy has significantly improved the outcome of patients with B cell malignancies. Regardless of this success, many patients still relapse with therapy-resistant disease, highlighting the need for the development of mAbs with higher capacity to induce programmed cell death. The so-called type II anti-CD20 mAbs (e.g., tositumomab) that trigger caspase-independent $B$ cell lymphoma cell death in vitro and show superior efficacy as compared with rituximab in eradicating target cells in mouse models are emerging as the next generation of therapeutic anti-CD20 mAbs. In this issue of the JCI, Ivanov and colleagues identify the lysosomal compartment as a target for type II mAbs (see the related article beginning on page 2143). These data encourage the further clinical development of type II mAbs as well as other lysosome-targeting drugs in the treatment of $B$ cell malignancies.

Caspase-mediated apoptosis is the main mechanism of action of most current anticancer treatments. Defects in apoptosis signaling pathways are, however, among the

Conflict of interest: The authors have declared that no conflict of interest exists.

Nonstandard abbreviations used: Ag, antigen.

Citation for this article: J. Clin. Invest. 119:2133-2136 (2009). doi:10.1172/JCI40259. major hallmarks of cancer, and apoptosisinducing therapies further select for highly apoptosis-refractory tumor cell clones (1). Accordingly, new strategies to kill cancer cells by nonapoptotic mechanisms have flourished during the past decade, and many mediators of alternate cell death pathways have been identified. Among them are the lysosomes with their large arsenal of proteolytic and lipolytic hydrolases $(2,3)$.
The lysosome: an emerging target for cancer therapy

Lysosomes function as cellular recycling and waste disposal units by degrading organelles and macromolecules delivered to the lysosomal compartment by autophagy, endocytosis, and phagocytosis (4). The degradation is performed by over 50 lysosomal hydrolases that can process all the major macromolecules of the cell to break down products available for metabolic reutilization. The lysosomal cell death pathways are characterized by partial lysosomal membrane permeabilization and the subsequent translocation of lysosomal hydrolases into the cytosol (2, 3). Once in the cytosol, lysosomal hydrolases, particularly cathepsin proteases, can trigger caspase-independent and BCL-2-insensitive cell death pathways even in highly apoptosisresistant cancer cells.

The potential of lysosomes as powerful "cell suicide bags" was recognized already in the 1950s by Christian de Duve, who received the Nobel prize for his discovery of 


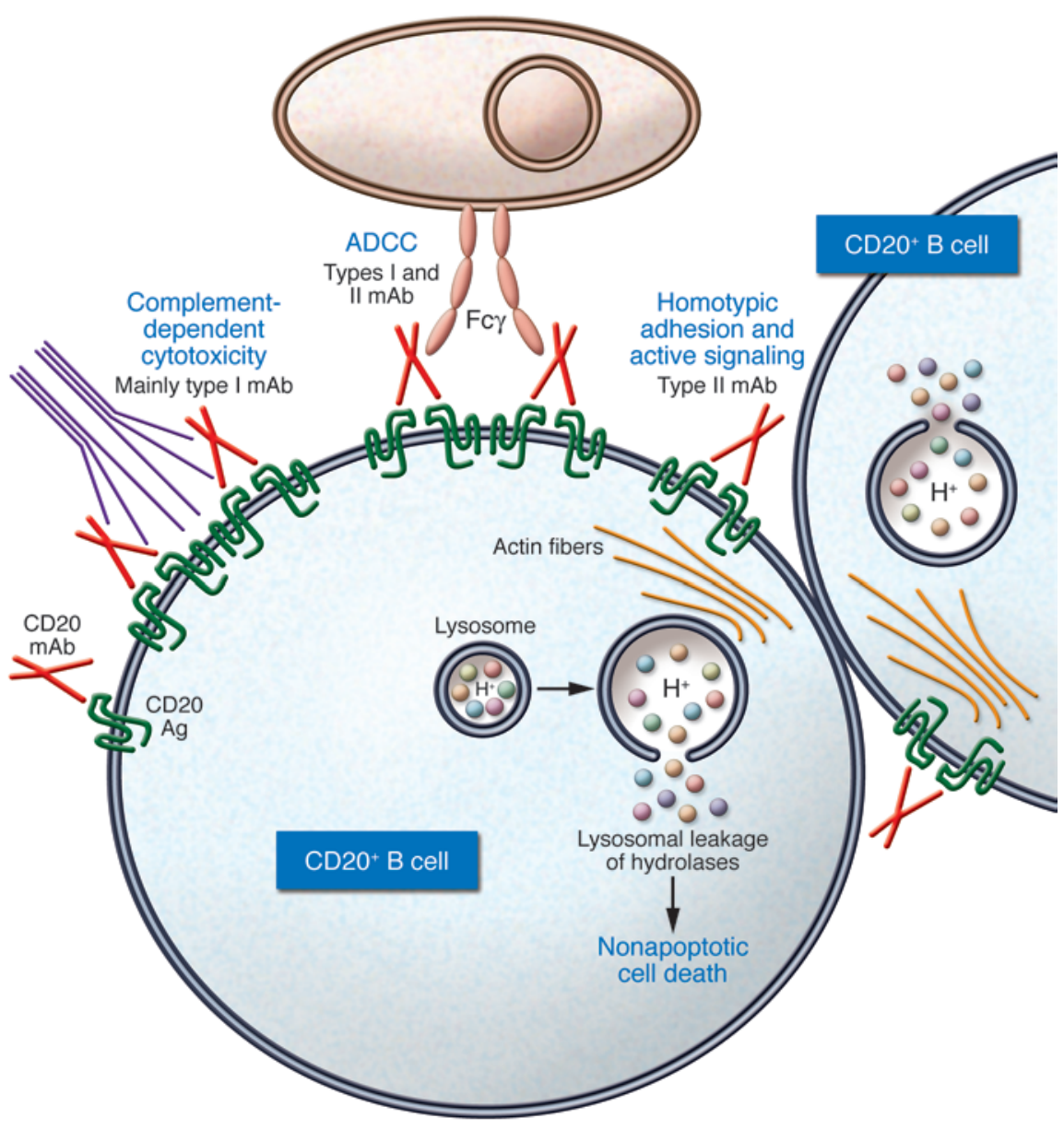

lysosomes. The further development of lysosome-targeting drugs for cancer treatment was, however, discouraged for many decades because of the presumption that they would kill all lysosome-bearing cells, i.e., all human cells except for mature red blood cells. The widespread apoptosis resistance in aggressive cancers together with recent discoveries indicating that the cancer-associated dramatic increase in lysosomal activity sensitizes cancer cell lysosomes to permeabilization has, however, revived interest in lysosometargeting drugs in cancer treatment (3).

\section{Rituximab has heralded a new era in cancer treatment}

Rituximab, a chimeric mAb targeting a B cell-specific CD20 surface antigen (Ag), was the first therapeutic $m A b$ approved for use in treating human malignancy by the US Food and Drug Administration. Over the last decade, its addition to conventional chemotherapy has greatly increased the response rates and survival times in patients with both aggressive and indolent B cell malignancies, including diffuse large
B cell lymphoma, follicular lymphoma, mantle cell lymphoma, and chronic lymphocytic leukemia (5-7). In spite of these achievements, a significant proportion of patients still relapse with a disease that has acquired resistance against immunochemotherapy. In particular, it has been demonstrated that tumors with defective apoptosis pathways due to the overexpression of the antiapoptotic protein MCL1 and transcriptional repressor BCL-6, constitutive activation of the NF- $\kappa \mathrm{B}$ pathway, or loss of tumor suppressor TP53 are less sensitive than other tumors to combination therapies with rituximab (8-11).

The efficacy of mAbs as anticancer agents may rely on several mechanisms. In addition to the Ag-independent IgG-induced pathways, i.e., Ab-dependent cellular cytotoxicity and complement-dependent cytotoxicity, mAbs may trigger a number of Ag-specific mechanisms that either inhibit cell growth or activate cell death pathways (Figure 1). Even though rituximab-mediated cross-linking of the CD20 Ag has been reported to activate the caspase-dependent mitochondrial

\section{Figure 1}

The effector mechanisms of type I and type II anti-CD20 mAbs. Whereas type I anti-CD20 mAbs induce cytotoxicity in B cells mainly via complement-dependent cytotoxicity and $\mathrm{Ab}$ dependent cellular cytotoxicity (ADCC), type II anti-CD20 mAbs are weaker at activating complement-dependent cytotoxicity. Instead, type II anti-CD20 mAbs are potent inducers of actin-dependent homotypic adhesions between $\mathrm{B}$ cells. In this issue of the $\mathrm{JCl}$, Ivanov and colleagues demonstrate that these adhesions are associated with localization of lysosomes to the proximity of cell-cell contacts, lysosomal swelling, and lysosomal membrane permeabilization (17). Importantly, the lysosomal hydrolases leaked into the cytosol can then induce nonapoptotic cell death even in highly apoptosis-resistant and rituximabresistant B cell malignancies. Thus, type II mAbs may provide new treatment options even in aggressive B cell tumors resistant to current therapies. apoptosis pathway in sensitive target cells in vitro, several lines of evidence suggest that its in vivo efficacy relies mainly on conventional mechanisms involving the complement and immune effector cells (12).

The clinical success of rituximab has led to a growing interest in mAb-based therapies in general and in those targeting CD20 in particular. The functional analysis of numerous existing anti-CD20 mAbs has allowed their division into at least two functionally separate groups based on their ability to redistribute CD20 molecules in the plasma membrane and activate various effector functions (13). The so-called type II anti-CD20 mAbs (such as tositumomab) are more potent in inducing programmed cell death than the type I anti-CD20 mAbs (such as rituximab) both as single agents and when combined with low-dose X-ray radiation (14). Importantly, the greater capacity of type II mAbs to induce programmed cell death in vitro correlates with their better efficacy in depleting malignant B cells in murine xenograft models as well as B cells in human CD20-transgenic mice $(15,16)$. 


\section{Lysosomes as targets for type II mAbs}

Based on the observation that $\mathrm{F}(\mathrm{ab}){ }_{2}$ fragments of type II but not those of type I antiCD20 mAbs provide substantial immunotherapy, it has been suggested that the greater efficacy of type II anti-CD20 mAbs relies on their ability to signal programmed cell death (15). Taking into consideration the highly apoptosis-resistant nature of many B cell malignancies, it is important to note that tositumomab induces a nonapoptotic cell death, which is independent of caspase activation and insensitive to the antiapoptotic action of BCL-2 (13). So far, the only clue to the nonapoptotic cytotoxic mechanism of action of tositumomab is its correlation with homotypic adhesion (aggregation) of the cells. In this issue of the JCI, Ivanov and colleagues shed further light on this mechanism (17). They provide ample data causally connecting tositumomab-induced cell death with actin reorganization, homotypic adhesion, and lysosomal membrane permeabilization. Using a variety of B cell lymphoma cell lines and a panel of primary samples from patients with chronic lymphocytic leukemia combined with pharmacological inhibitors of actin polymerization, they convincingly demonstrate that both the homotypic adhesion and cell death induction depend on actin dynamics. Using the Raji B cell lymphoma cell line as a model system, they then take a closer look at the cellular and molecular changes induced by tositumomab. In this model system, they identify lysosomes as effectors of tositumomab-induced cytotoxicity. They demonstrate that tositumomab triggers an initial increase in the volume of the lysosomal compartment followed by the collapse of this compartment prior to plasma membrane permeabilization. And more importantly, the causative link between lysosomal leakage and cell death is provided by the remarkable cytoprotection brought about by the otherwise subtoxic pharmacological inhibitors of cysteine cathepsins (Z-PheGly-NHO-Bz-pOM) and a vacuolar proton pump (concanamycin A and bafilomycin).

Intriguingly, the data by Ivanov et al. show that type II mAbs directed against the MHC class II cell surface receptor HLA-DR also trigger actin-dependent homotypic adhesion and the lysosomal cell death pathway, whereas type I anti-CD20 mAbs do not (17). These data suggest that both the cell adhesion and the lysosomal cell death are dependent on the mode of interaction between the Ag and the $\mathrm{mAb}$ rather than on the nature of the Ag targeted. In this context, it would be interesting to determine whether this phenomenon also translates to type II mAbs recognizing other target molecules, such as CD99, whose cross-linking by $\mathrm{mAb}$ has been reported to induce caspase-independent cell death (18).

It remains to be revealed how type II mAbs trigger lysosomal destabilization. Based on the dependence of cell death on actin dynamics, the correlation between intercellular membrane exchange and cell death, the localization of disrupted lysosomes and leaked cathepsins into the areas of cell-cell contact, and the dramatic swelling of the lysosomal compartment, it is tempting to speculate that homotypic adhesions may induce an uncontrolled endocytosis response resulting in an enlargement of the lysosomal compartment. Interestingly, the cathepsin B staining pattern observed in the treated cells suggests that tositumomab dramatically increases the expression of this lysosomal hydrolase (17), whose increased activity can destabilize lysosomal membranes by a mechanism involving degradation of lysosomal membrane proteins (19). More quantitative experiments addressing cathepsin activity as well as the analysis of endocytic activity would, however, be needed to test this hypothesis, which was not addressed by the authors.

\section{Future perspectives}

The current study by Ivanov and coworkers demonstrates that type II mAbs such as tositumomab induce the nonapoptotic lysosomal cell death pathway (17). Given that many lymphomas have fundamental defects in both mitochondrial and death receptor signaling pathways and that these apoptotic defects specifically characterize the most aggressive and rituximab-resistant tumors, type II mAbs may provide new treatment options in aggressive $B$ cell tumors. Indeed, tositumomab treatment has been demonstrated to result in tumor regression in refractory/relapsed non-Hodgkin lymphoma patients and even more remarkable responses have been reported for the ${ }^{131} \mathrm{I}$-conjugated version of tositumomab (Bexxar) that shows efficacy even in cases in which rituximabbased therapy has failed $(20,21)$. It will be of importance to investigate in future clinical trials whether the superiority of the unconjugated type II mAbs compared with type I mAbs seen in the experimental models also holds true in patients (14-16).
Type II mAbs may also be beneficial as first-line therapy in combination with existent chemotherapeutic regimens, since it may prohibit the development of resistant cells with apoptosis defects. In light of this new data on the lysosomal involvement in the cytotoxicity induced by type II mAbs, combination therapies with agents further sensitizing cells to lysosomal destabilization (such as microtubule-disturbing drugs vincristine and paciltaxel) as well as with those inducing lysosomal destabilization (such as siramesine) should be experimentally tested $(22,23)$. Finally, it can be speculated whether regimens that cause activation of the lysosomal cell death pathway may also be effective in patients with inherited diseases caused by defects in proapoptotic genes such as TP53 (Li-Fraumeni syndrome) and FAS (autoimmune lymphoproliferative syndrome).

\section{Acknowledgments}

The research in the authors' laboratories has been supported by the Danish Cancer Society, the Danish National Research Foundation, the Danish Medical Research Council, European Commission FP7 (APO-SYS), the Meyer Foundation, the Lundbeck Foundation, and the Novo Nordisk Foundation.

Address correspondence to: Marja Jäättelä, Apoptosis Department, Institute for Cancer Biology, Danish Cancer Society, Strandboulevarden 49, DK-2100 Copenhagen, Denmark. Phone: 45-35257318; Fax: 4535257721; E-mail:mj@cancer.dk.

1. Hanahan, D., and Weinberg, R.A. 2000. The hallmarks of cancer. Cell. 100:57-70.

2. Kroemer, G., and Jäättelä, M. 2005. Lysosomes and autophagy in cell death control. Nat. Rev. Cancer. 5:886-897.

3. Kirkegaard, T., and Jäättelä, M. 2009. Lysosomal involvement in cell death and cancer. Biochim. Biophys. Acta. 1793:746-754.

4. Luzio, J.P., Pryor, P.R., and Bright, N.A. 2007. Lysosomes: fusion and function. Nat. Rev. Mol. Cell Biol. 8:622-632.

5. Michallet, A.S., and Coiffier, B. 2009. Recent developments in the treatment of aggressive non-Hodgkin lymphoma. Blood Rev. 23:11-23.

6. Quintas-Cardama, A., and O'Brien, S. 2009. Targeted therapy for chronic lymphocytic leukemia. Target Oncol. 4:11-21.

7. Fanale, M.A., and Younes, A. 2007. Monoclonal antibodies in the treatment of non-Hodgkin's lymphoma. Drugs. 67:333-350.

8. Winter, J.N., et al. 2006. Prognostic significance of Bcl-6 protein expression in DLBCL treated with CHOP or R-CHOP: a prospective correlative study. Blood. 107:4207-4213.

9. Awan, F.T., et al. 2009. Mcl-1 expression predicts progression-free survival in chronic lymphocytic leukemia patients treated with pentostatin, cyclophosphamide, and rituximab. Blood. 113:535-537.

10. Lenz, G., et al. 2008. Stromal gene signatures in large- 
B-cell lymphomas. N. Engl. J. Med. 359:2313-2323.

11. Byrd, J.C., et al. 2006. Select high-risk genetic features predict earlier progression following chemoimmunotherapy with fludarabine and rituximab in chronic lymphocytic leukemia: justification for risk-adapted therapy. J. Clin. Oncol. 24:437-443.

12. Johnson, P., and Glennie, M. 2003. The mechanisms of action of rituximab in the elimination of tumor cells. Semin. Oncol. 30:3-8.

13. Chan, H.T., et al. 2003. CD20-induced lymphoma cell death is independent of both caspases and its redistribution into triton $\mathrm{X}-100$ insoluble membrane rafts. Cancer Res. 63:5480-5489.

14. Ivanov, A., Krysov, S., Cragg, M.S., and Illidge, T. 2008. Radiation therapy with tositumomab (B1) antiCD20 monoclonal antibody initiates extracellular signal-regulated kinase/mitogen-activated protein kinase-dependent cell death that overcomes resis- tance to apoptosis. Clin. Cancer Res. 14:4925-4934.

15. Cragg, M.S., and Glennie, M.J. 2004. Antibody specificity controls in vivo effector mechanisms of anti-CD20 reagents. Blood. 103:2738-2743.

16. Beers, S.A., et al. 2008. Type II (tositumomab) antiCD20 monoclonal antibody out performs type I (rituximab-like) reagents in B-cell depletion regardless of complement activation. Blood. 112:4170-4177.

17. Ivanov, A., et al. 2009. Monoclonal antibodies directed to CD20 and HLA-DR can elicit homotypic adhesion followed by lysosome-mediated cell death in human lymphoma and leukemia cells. J. Clin. Invest. 119:2143-2159.

18. Cerisano, V., et al. 2004. Molecular mechanisms of CD99-induced caspase-independent cell death and cell-cell adhesion in Ewing's sarcoma cells: actin and zyxin as key intracellular mediators. Oncogene. 23:5664-5674

19. Fehrenbacher, N., et al. 2008. Sensitization to the lysosomal cell death pathway by oncogene-induced down-regulation of lysosome-associated membrane proteins 1 and 2. Cancer Res. 68:6623-6633.

20. Davis, T.A., et al. 2004. The radioisotope contributes significantly to the activity of radioimmunotherapy. Clin. Cancer Res. 10:7792-7798.

21. Horning, S.J., et al. 2005. Efficacy and safety of tositumomab and iodine-131 tositumomab (Bexxar) in B-cell lymphoma, progressive after rituximab. J. Clin. Oncol. 23:712-719.

22. Ostenfeld, M.S., et al. 2008. Anti-cancer agent siramesine is a lysosomotropic detergent that induces cytoprotective autophagosome accumulation. Autophagy. 4:487-499.

23. Groth-Pedersen, L., Ostenfeld, M., Høyer-Hansen, M., Nylandsted, J., and Jäättelä, M. 2007. Vincristine induces dramatic lysosomal changes and sensitizes cancer cells to lysosome destabilizing siramesine. Cancer Res. 67:2217-2225.

\section{EBAG9 tempers lymphocyte killing activity}

Gaël Ménasché ${ }^{1,2}$ and Geneviève de Saint Basile ${ }^{1,2}$

1INSERM U768, Paris, France. ²Faculté de Médecine, Université Paris Descartes, Paris, France.

\begin{abstract}
The cytotoxic activity of lymphocytes is crucial for immune surveillance and homeostasis. Several independent, naturally occurring genetic models characterized by defects in granule trafficking or exocytosis have helped to decipher the multiple steps and molecules that regulate the cytotoxic process. The study by Rüder and colleagues in this issue of the JCI shows that an engineered absence of EBAG9, previously reported as a tumor-associated antigen, enhances cytotoxic activity of CTLs but not NK cells, likely acting on the endosomal-lysosomal trafficking of the cytotoxic effectors (see the related article beginning on page 2184). This finding adds a new piece to the puzzle of complex mechanisms that tightly regulate the capacity of the cytotoxic response and suggests a new target to negatively modulate CTL responsiveness.
\end{abstract}

CTLs and NK cells (collectively known as "cytotoxic lymphocytes") are major players in the body's defense against viral infection and cancer via their ability to seek out and kill infected or tumorigenic cells. Although CTLs are activated by specific antigen recognition, the cytotoxic activity of NK cells is initiated by specific activating receptors or combinations thereof and is inhibited by self MHC class I recognition. Once cyto-

Conflict of interest: The authors have declared that no conflict of interest exists.

Nonstandard abbreviations used: AP1, adaptor protein 1 ; BLOC-1, biogenesis of lysosome-related organelles complex 1; EBAG9, estrogen receptor-binding fragment-associated antigen 9; ESCRT, endosomal sorting complex required for transport; HLH, hemophagocytic lymphohistiocytosis; HPS2, HermanskyPudlak syndrome type 2; IS, immunological synapse; LAMP, lysosomal-associated membrane protein; LCMV, lymphocytic choriomeningitis virus; LRO, lysosomerelated organelle; LYST, lysosomal trafficking regulator. Citation for this article: J. Clin. Invest. 119:2136-2140 (2009). doi:10.1172/JCI40270. toxic cells have recognized their targets and formed a conjugate, the trafficking of granule components, including perforin and granzymes, to the immunological synapse (IS) between the cell and its target, leads to the delivery of perforin and granzymes into the target cells and subsequent target destruction through apoptosis $(1,2)$.

Studies of natural or engineered mutants involving cytotoxic function tell us a great deal about the in vivo function of this pathway. So far, only genetic diseases leading to the loss of cytotoxic function have been reported (3). These studies have revealed the tremendous importance of this cytotoxic pathway in immune homeostasis. Congenital defects that lead to either impaired perforin function or its dysregulated release result in the specific severe condition hemophagocytic lymphohistiocytosis (HLH) syndrome. This syndrome stems from the inability of activated cytotoxic cells to clear antigen- presenting targets. The failure to clear antigen causes unremitting polyclonal $\mathrm{CD}^{+} \mathrm{T}$ cell expansion, activation, and infiltration of visceral organs associated with macrophage activation, with the deleterious release of multiple inflammatory cytokines including IFN- $\gamma(3,4)$.

In addition, the studies of these natural mutants both in humans and in mice have contributed to the characterization of critical effectors of the cytotoxic machinery and their function. They also provided evidence of the exquisite regulation of this process, which occurs in successive steps following cell activation. For instance, studies of Griscelli syndrome type 2, a rare condition characterized by partial albinism and occurrence of an HLH syndrome (5), and familial HLH type 3 (FHL3) (6) have shown the critical role of two proteins, the small GTPase Rab27a and the priming factor Munc13-4, at a late step of the secretory pathway. Rab27a regulates the tethering of mature cytotoxic granules that have polarized at the IS, whereas Munc13-4 primes the docked cytotoxic granules before their fusion with the plasma membrane at the IS. Other proteins, for which their defect impairs lymphocyte cytotoxic activity, likely regulate an upstream step in the cytotoxic pathway. They include adapter protein 3 (AP3), which is deficient in Hermansky-Pudlak syndrome type 2 (HPS2) $(7,8)$, and the lysosomal trafficking regulator (LYST) protein, which is deficient in Chediak-Higashi syndrome $(9,10)$. Their 